\title{
Failure probability estimation of overhead transmission lines considering the spatial and temporal variation in severe weather
}

\author{
Shuai YANG ${ }^{1}$, Wenjun ZHOU ${ }^{1}$, Shiyang ZHU $^{2}$, Le WANG ${ }^{2}$, \\ Lisha $\mathrm{YE}^{3}$, Xuezhi XIA ${ }^{1}$, Han LI ${ }^{1}$
}

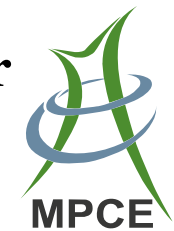

\begin{abstract}
Estimation for weather-related failure probability of overhead transmission lines is essential in the reliability assessment of a power system. This paper analyzes the outage and weather data of $110 \mathrm{kV}$ overhead transmission lines in the Guangxi Zhuang Autonomous Region of China during 2011-2014. The result reveals obvious uneven distributions of outage events for time and space due to the spatial and temporal variation of severe weather. Based on the results, an estimation method is proposed in this paper. Split and aggregation is used to smooth the
\end{abstract}

CrossCheck date: 15 November 2017

Received: 28 December 2016/Accepted: 15 November 2017/ Published online: 10 March 2018

(C) The Author(s) 2018. This article is an open access publication

Shuai YANG

ys3254@163.com

Wenjun ZHOU

wjzhou@whu.edu.cn

Shiyang ZHU

juiceyoung@163.com

Le WANG

happywle@163.com

Lisha YE

yls91126@163.com

Xuezhi XIA

792217148@qq.com

Han LI

golden.silver@163.com

1 School of Electrical Engineering, Wuhan University, Wuhan 430072, China

2 Electric Power Research Institute, Guangxi Power Grid Co., Ltd, Nanning 530023, China

3 Hubei Electric Engineering Corporation, Wuhan 430040, China outage and weather data. The poisson model is adopted in our method to investigate the statistic characteristics of transmission line outage events. Regression analysis is applied to obtain the correlation between the weather intensity and history failure rate. Furthermore the method proposed is validated against the empirical outage data.

Keywords Severe weather, Transmission line, Poisson model, Failure probability, Regression analysis

\section{Introduction}

Overhead transmission lines are exposed to nature and are vulnerable to severe weather, such as lighting, storms and hurricanes and so on [1-4]. Operational security of overhead transmission lines has a close relationship with the external meteorological conditions. Weather factors need to be taken into consideration for a reliability assessment of overhead transmission lines, which is important for economic- security decision making [5-7]. Failure probability of overhead transmission lines is essential in applying an appropriate reliability assessment method.

The Markov model [8] is widely used to estimate the failure rate of overhead transmission lines. Considering the intensity of the weather factors, the Markov model is being developed as a two-state, three-state, and multi-state weather model and so on [9-11]. Chong Wang presents a Markov-based deterioration model for transmission components [12]. Ali Arab et al. use partially observable Markov decision processes to develop a generic model for integrated condition-based maintenance of the electric power systems infrastructure [13]. Yong Liu and Chanan Singh introduced a DC-OPF based Markov cut-set method 
to evaluate composite power system reliability considering weather effects [14]. These models are primarily suitable for relatively long-term applications. There are both temporal and spatial variations of weather factors $[15,16]$. Estimation of the weather influence on transmission lines is required to capture the stochastic behavior of the weather [17].

The Monte Carlo method is applied to simulate the stochastic behavior after the modeling of complex system operational conditions [18]. Billinton and Wenyuan [19] used the two-state weather model and the Monte Carlo sampling technique to incorporate the variable weather conditions in a composite analysis. Mathaios Panteli and Pierluigi Mancarella introduced a novel sequential MonteCarlo-based time-series simulation model to assess power system resilience [20]. The Monte Carlo simulation depends on random experiments and its convergence may require acceleration by using other techniques [15].

In order to establish a simple relationship between failure probability and weather factors, statistical analysis and the artificial intelligence method are also adopted [21]. For example, the noisy OR-gate model, regression model and fuzzy model are proposed in many literatures [22-25]. In these methods, the average failure rate model is still used after data splitting and aggregation. Weather can affect all the transmission lines in a certain area. The outage event in this area has a random nature and statistical characteristic, which is ignored in the average failure rate model. The poisson model is proposed in [26] to consider the statistical characteristics of transmission line outage events. But the model is not fully described and not validated against empirical outage data.

In order to determine the distribution of outage events on time and space, outage and weather data of an $110 \mathrm{kV}$ overhead transmission line from the Guangxi Zhuang Autonomous Region of China during 2011-2014 are analyzed in this paper. A failure probability estimation method for overhead transmission lines is proposed in this paper considering lightning and wind. Statistical characteristics of an outage event are investigated based on the poisson model. The relationship between the poisson model parameter and history failure rate is presented. Furthermore the model is validated against the empirical outage data.

\section{Outage events of transmission lines in Guangxi Zhuang Autonomous Region}

Outage events of $110 \mathrm{kV}$ transmission lines in Guangxi Zhuang Autonomous Region of China during 2012-2014 are classified as shown in Fig. 1. Weather-related outage events account for $78.09 \%$ in total. Lighting and wind are

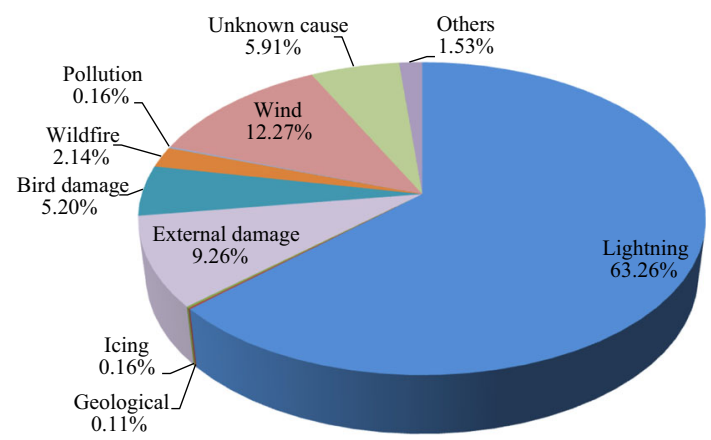

Fig. 1 Proportion of transmission line outage causes

the two main severe weather factors, which are taken into consideration in the following analysis.

Due to the temporal and spatial variation of weather factors, the distribution of weather-related outage events is uneven for time and space. As shown in Figs. 2 and 3, lightning-related outages in Fangchenggang city, Wuzhou city and Guilin city are the top three, accounting for $14.55 \%, 12.29 \%$ and $11.43 \%$ respectively. Strong wind primarily affects the coastal areas, such as Beihai city, Qinzhou city and Fangchengang city and the outages account for $35.71 \%, 22.77 \%$ and $21.88 \%$ respectively. The
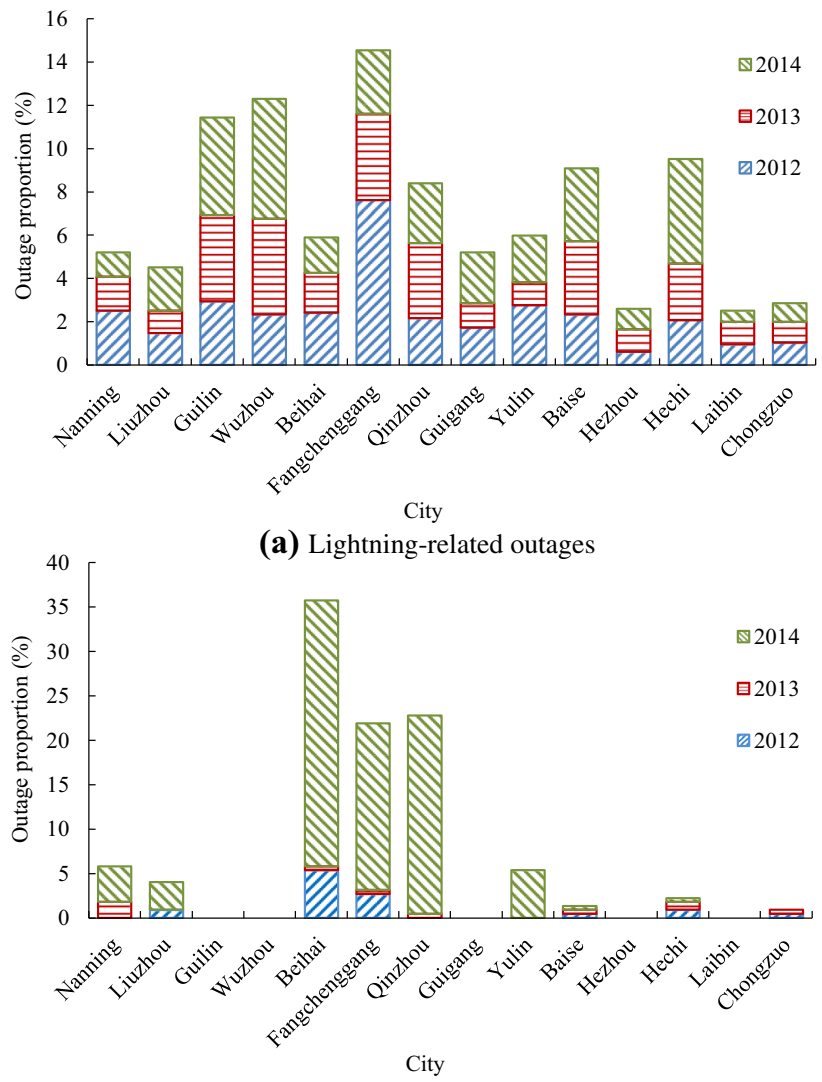

(b) Wind-related outages

Fig. 2 Spatial variation of weather-related transmission line outages 


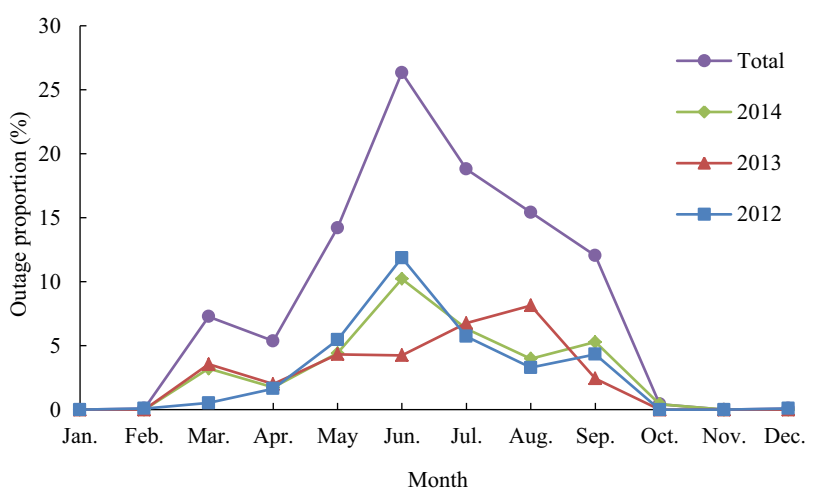

(a) Lightning-related outages

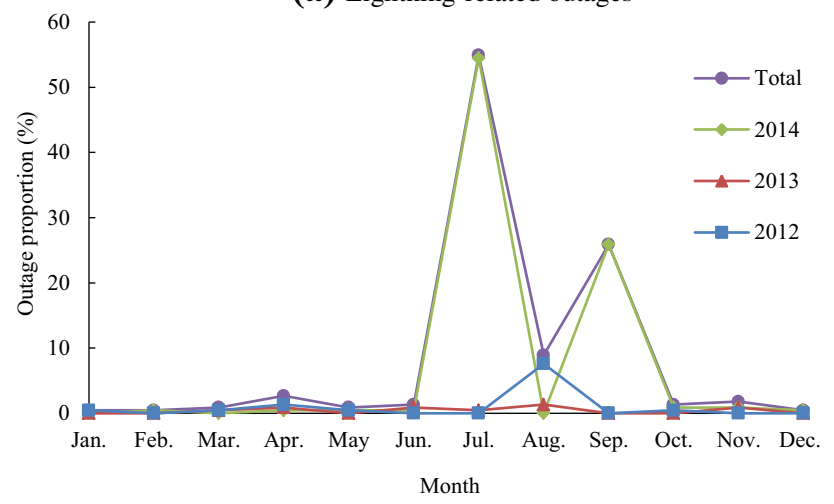

(b) Wind-related outages

Fig. 3 Temporal variation of weather-related transmission line outages

monthly variation of outage events is closely related to the activities of lightning and wind. It is primarily concentrated from May to September, which accounts for $78.59 \%$ of the whole year. Outages induced by wind are primarily concentrated from June to October and are related to typhoon activity. Because of the uneven distribution of weather factors for time and space, there are obvious differences for transmission line outages between various regions in each month. It is necessary to deal with the temporal and spatial variations of weather factors and outage events to make short-term predictions.

\section{Estimation for transmission line failure probability}

\subsection{Temporal and spatial split of weather and outage data}

According to the weather and outage data of transmission lines, appropriate weather factors are determined in the method and denoted as $w_{1}, w_{2}, \ldots, w_{p}$ respectively. Due to the temporal and spatial variations of weather factors, temporal and spatial splits are needed. Distribution of weather factors is uneven over a vast area, such as a province. A transmission line might be in multiple areas with a different meteorological environment. A spatial split is the division of weather areas. Assuming that the meteorological environment in each weather area is consistent, the divided weather areas are denoted as $\mathrm{WA}_{1}, \mathrm{WA}_{2}, \ldots, \mathrm{WA}_{q}$ respectively. According to the data from the meteorological department, the weather area is divided the same as the administrative area. There are several lines in one weather area, which are in the same meteorological environment. All transmission lines with the same voltage level are taken as the basic samples for the estimation of one weather area. Temporal treatment of weather data contains both split and aggregation. First weather and outage data are split by a step of one day and the weather data is classified into several intensities. Based on the intensity classification, the weather and outage data with the same intensity are aggregated. Both temporal and spatial aggregation result in data smoothing and provides more meaningful patterns and it converts the time distribution of the weather factors to the intensity distribution. There are several weather factors in one area and the combination of different weather factors is defined as a weather block. As the weather factor $w_{1}$ and $w_{2}$ in a weather area is at level $\mathrm{k}$ and $\mathrm{m}$ respectively, the weather block is defined as $\mathrm{WB}_{k m}$. The outage data is divided according to the classification of the weather block.

\subsection{Probability distribution of a transmission line failure based on poisson model}

In each weather area, the outage events per day resulting from the condition of each weather block can be considered as discrete rare events. The failure probability satisfies the poisson model and its expression is:

$P(X=k)=e^{-\theta} \theta^{k} / k ! \quad k=0,1, \ldots$

where $X$ is the occurrence number of the random event.

It is assumed that there are $n$ lines in the weather area $\mathrm{WA}_{i}$, and the line lengths are $l_{1}, l_{2}, \ldots, l_{n}$ respectively. $X$ is the line outage event per unit time resulting from the condition of the weather block $\mathrm{WB}_{k m}$. Outage event per unit time of each transmission line is $X_{1}, X_{2}, \ldots, X_{n}$ respectively, and they are independent events. The observation value of each event is $x_{1}, x_{2}, \ldots, x_{n}$ respectively. $X$ follows the poisson model, and the failure probability of the transmission line per unit time is:

$P(X \geq 1)=1-e^{-\theta}$

According to the maximum likelihood estimation method, the likelihood function is: 
$L(\theta)=\prod_{i=1}^{n} p\left(x_{i}, \theta\right)=\prod_{i=1}^{n} e^{-\theta} \frac{\theta^{x_{i}}}{x_{i} !}=e^{-n \theta} \theta^{\sum_{i=1}^{n} x_{i}}\left(\prod_{i=1}^{n} x_{i} !\right)^{-1}$

$\hat{\theta}=\frac{1}{n} \sum_{i=1}^{n} x_{i}$

$T_{W B}$ is defined to denote the days of the weather block and $z_{1}, z_{2}, \ldots, z_{n}$ denote the outage times of each transmission line occurring in the duration of the historical data respectively. Because the repairing time is too small it can be neglected and the failure rate is approximately represented as the failure frequency in unit length and unit time as follows:

$$
\begin{aligned}
\lambda^{*} & =\left(T_{W B} \sum_{i=1}^{n} l_{i}\right)^{-1} \sum_{i=1}^{n} z_{i}=\left(\frac{1}{n} \sum_{i=1}^{n} x_{i}\right)\left(\frac{1}{n} \sum_{i=1}^{n} l_{i}\right)^{-1} \\
& =\frac{1}{n \times \bar{L}} \sum_{i=1}^{n} x_{i}=\frac{\hat{\theta}}{\bar{L}}
\end{aligned}
$$

$\bar{L}=\frac{1}{n} \sum_{i=1}^{n} l_{i}$

where $L$ is the average length of the transmission lines in the weather area.

Historical outage data of transmission lines can be used to calculate the parameter of the poisson model. According to historical weather and outage data, the relationship between the historical failure rate and weather factors can be established. Based on the poisson model, the failure probability of the transmission line can be predicted using the weather forecast data.

\subsection{Regression analysis}

Regression analysis is used in this paper to establish the relationship between the historical failure rate $\lambda^{*}$ and weather factors $w_{1}, w_{2}, \ldots, w_{p}$. Because $\lambda^{*}$ is too small to be calculated, $\lambda^{\prime}$ is defined to denote the logarithm of $\lambda^{*}$, which is shown as follows:

$\lambda^{\prime}=\ln \left(\lambda^{*}\right)=\ln \left(\left(T_{W B} \sum_{i=1}^{n} l_{i}\right)^{-1} \sum_{i=1}^{n} z_{i}\right)$

The regression model of $\lambda^{\prime}$ and weather factors can be expressed in matrix form as follows:

$\lambda^{\prime}=\boldsymbol{B} \boldsymbol{W}$

where $\boldsymbol{B}=\left[b_{0}, b_{1}, b_{2}, \ldots, b_{p}\right]$ and it is the regression coefficient matrix; $\boldsymbol{W}$ is the weather factor matrix.
The least square method is used to obtain the regression coefficient matrix. Furthermore, the significance test and applicability test are adopted to test the regression equation. Then the regression equation can be used to estimate.

\subsection{Failure probability of transmission lines}

If the average line length in a weather area is known, the outage time per line in unit time can be obtained:

$\bar{\lambda}=\bar{L} e^{B W}=\frac{1}{n} \sum_{i=1}^{n} x_{i}=\hat{\theta}$

Failure probability of transmission line in unit time can be calculated as follows:

$P=1-e^{-\bar{\lambda}}$

If a line passes through several weather areas, the nonfailure probability " $1-P$ " is the product of " $1-P_{\mathrm{WA}_{q}}$ " which means the non-failure probability of the line in each weather area. The total failure probability is calculated as follows:

$1-P=\left(1-P_{\mathrm{WA}_{1}}\right)\left(1-P_{\mathrm{WA}_{2}}\right) \cdots\left(1-P_{\mathrm{WA}_{q}}\right)$

Based on the above principles, the following steps are used to make a concrete calculation as shown in Fig. 4.

1) Determine main weather factors by analyzing the historical outage and weather data. Weather and outage data is classified according to the division of the weather area.

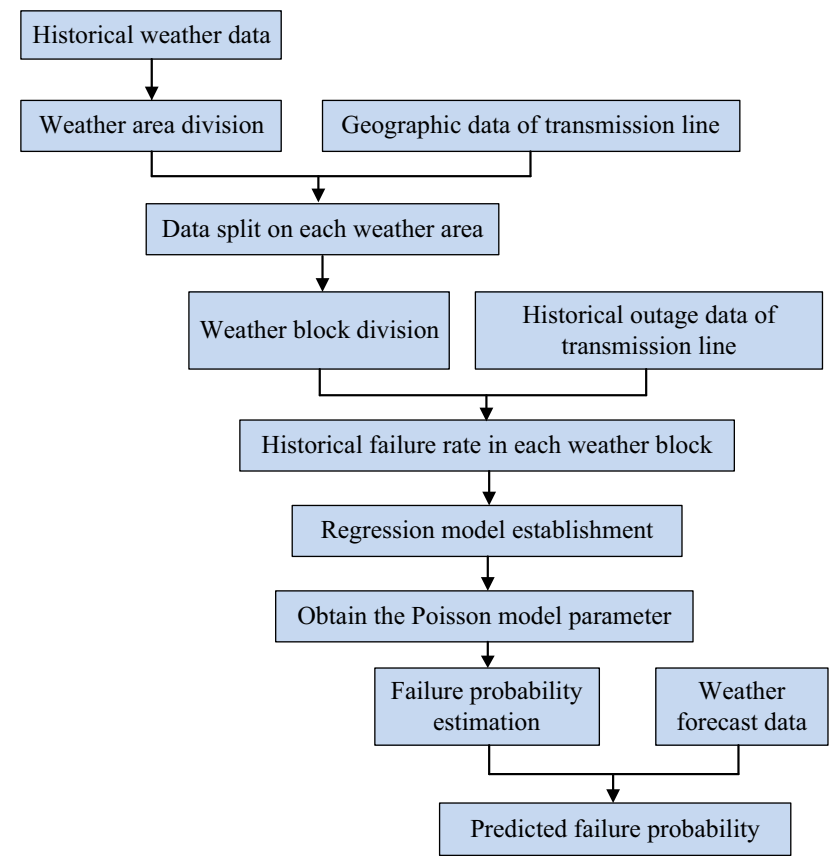

Fig. 4 Flow chart of estimation method proposed 
2) Classify the weather intensity and establish the weather block.

3) Count the outage number in different weather blocks and calculate the historical failure rate of the transmission line.

4) Establish the regression model of the historical failure rate and weather intensity and select the suitable regression model via the hypothesis test.

5) Obtain the poisson model parameter based on the historical outage and weather data.

6) Calculate the failure probability of the transmission line with the weather forecast data.

\section{Example}

\subsection{Temporal and spatial split of historical data}

According to the weather and outage data of the $110 \mathrm{kV}$ transmission line in the Guangxi Zhuang Autonomous Region of China during 2012-2014, two parameters denoted as $w_{1}$ and $w_{2}$ are chosen as the lightning density and maximum wind speed per day. The lightning density data is obtained from LLS [27] and the wind speed data is the instantaneous value obtained on the ground from the meteorological service of Guangxi. Taking Fangchenggang city with the most outages for example, the weather area of the whole city is named as $\mathrm{WA}_{0}$. Four counties in its jurisdiction, Gangkou, Fangcheng, Shangsi and Dongxin, are named as $\mathrm{WA}_{1}, \mathrm{WA}_{2}, \mathrm{WA}_{3}$ and $\mathrm{WA}_{4}$ respectively. Gangkou and Dongxin are next to the sea, and Shangsi is farthest from the seaside.

After dividing the weather area, lightning density and maximum wind speed of each weather area are collected and the weather intensity is classified into a weather block

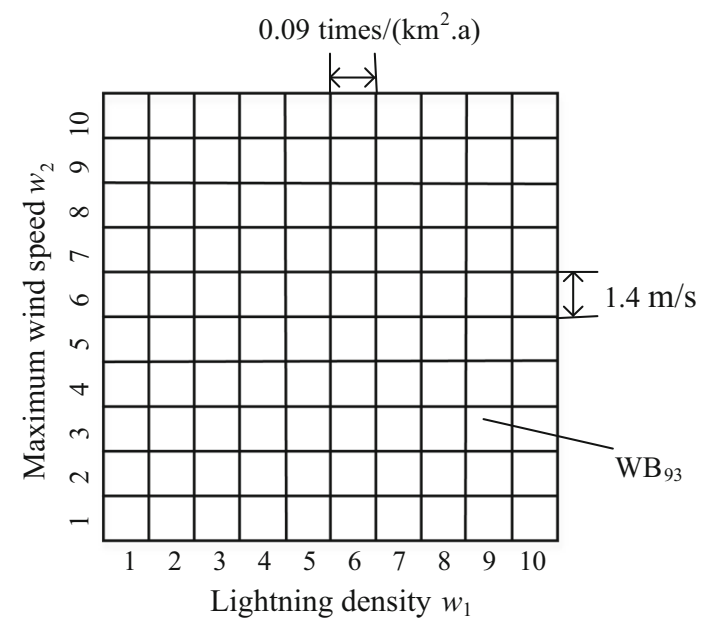

as shown in Fig. 5. The number of days in which lightning density is more than 0.9 times $/\left(\mathrm{km}^{2} \cdot \mathrm{a}\right)$ accounts for $0.4 \%$ in total days. Therefore, this paper focuses on the analysis of the outages with a lightning density between $0-0.9$ times/ $\left(\mathrm{km}^{2} \cdot \mathrm{a}\right)$. Lightning density is divided into 10 levels with an interval of 0.09 times $/\left(\mathrm{km}^{2} \cdot \mathrm{a}\right)$, named as $1-10$. The number of days in which the maximum wind speed is more than 14 $\mathrm{m} / \mathrm{s}$ represents $0.16 \%$, so the outages with the maximum wind speed between $0-14 \mathrm{~m} / \mathrm{s}$ are analyzed in this paper. Maximum wind speed is divided into 10 levels with an interval of $1.4 \mathrm{~m} / \mathrm{s}$, named as $1-10$. Combined with the outage data, statistics of the outage numbers at different weather blocks are obtained for subsequent calculation.

\subsection{Historical failure rate of the transmission line}

According to the third step of the calculation process, the outage numbers at different weather blocks are counted. The historical failure rate of the transmission line is calculated. The data during January 2012 to July 2014 is taken to establish the estimation model and the data during August 2014 to December 2014 is taken to verify the model.

Taking $\mathrm{WA}_{2}$ as an example, the total outage numbers and day numbers of each weather block are shown in Fig. 6a and b respectively. According to (7), historic failure rate in each weather block is shown in Fig. 6c. The day number with the lowest weather intensity is generally more than that with the highest weather intensity. Because the occurrence probability of severe weather is low, the outage number under strong weather intensity could be small. But the stronger the weather intensity is, the higher the failure rate will be. The historical failure rate in other weather areas is roughly the same as that of $\mathrm{WA}_{2}$.

\subsection{Estimation and verification of model}

According to the historical failure rate and weather intensity in each weather block, the regression model of $\lambda^{\prime}$ and also the weather factors is established. Comparing the hypothesis test result of multiple regression models, the polynomial regression model in (12) to (16) has the highest goodness of fit. The value of $p$ in the $f$-test is less than 0.01 and the value of $p$ in the $t$-test is less than 0.05 . In the applicability test, the residuals are all subject to the normal distribution and there is no difference and self-correlation. The regression results are significant.

$$
\begin{aligned}
\lambda_{\mathrm{WA}_{0}}^{\prime}= & -13.2719+2.8091 w_{1}-0.2515 w_{1} w_{2}-0.1438 w_{1}^{2} \\
& +0.0963 w_{2}^{2}
\end{aligned}
$$

Fig. 5 Division of weather block 


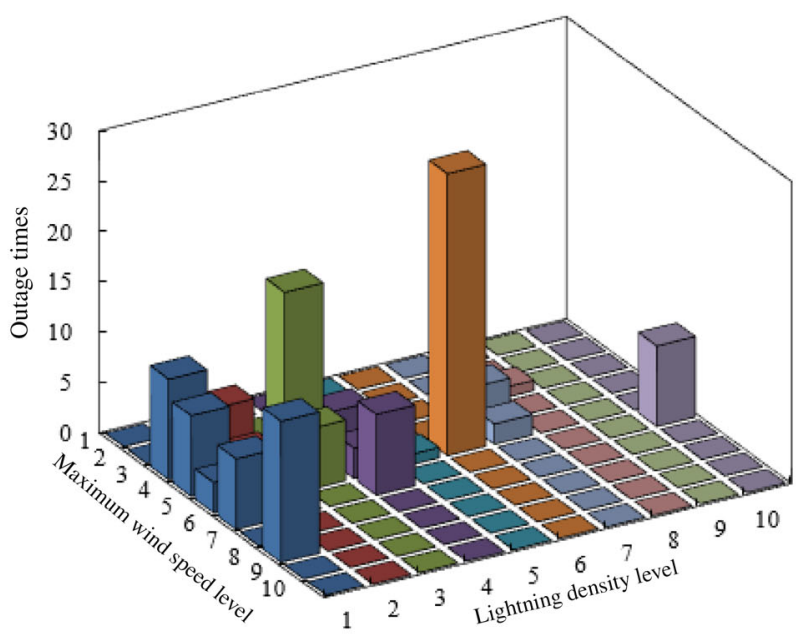

(a) Outage times

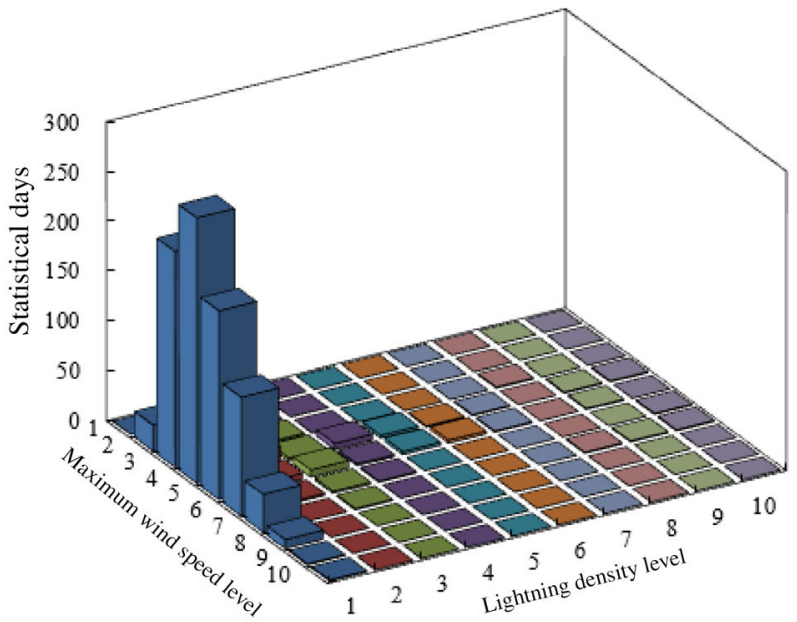

(b) Statistical days

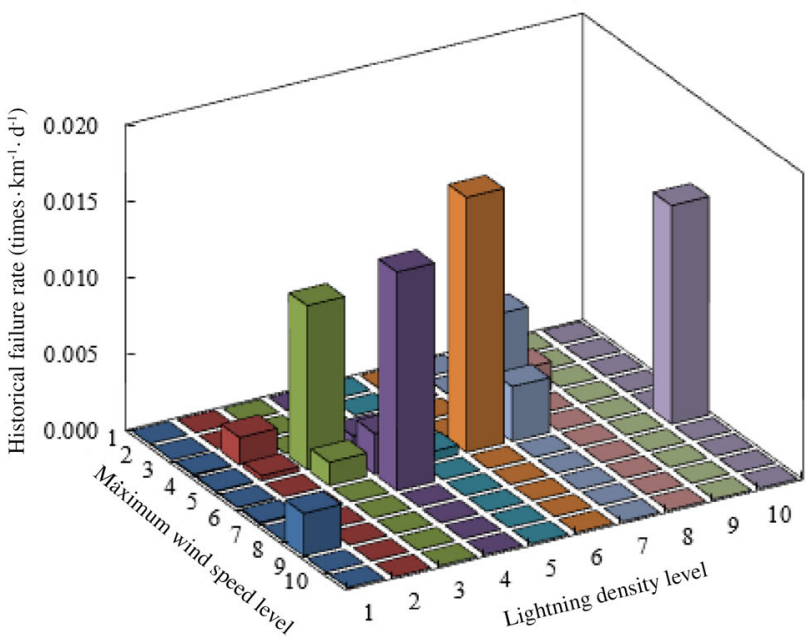

(c) Historical failure rate

Fig. 6 Historical statistics of transmission line outages in different weather blocks of $\mathrm{WA}_{2}$

$$
\begin{aligned}
\lambda_{\mathrm{WA}_{1}}^{\prime}= & -13.9225+3.1331 w_{1}-0.0791 w_{1} w_{2}-0.2645 w_{1}^{2} \\
& +0.0727 w_{2}^{2}
\end{aligned}
$$

$$
\begin{aligned}
\lambda_{\mathrm{WA}_{2}}^{\prime}= & -11.9084+1.6476 w_{1}-0.0387 w_{1} w_{2}-0.1069 w_{1}^{2} \\
& +0.0595 w_{2}^{2}
\end{aligned}
$$

$$
\begin{aligned}
\lambda_{\mathrm{WA}_{3}}^{\prime}= & -11.8423+1.0980 w_{1}+0.2043 w_{1} w_{2}-0.2692 w_{1}^{2} \\
& +0.0515 w_{2}^{2} \\
\lambda_{\mathrm{WA}_{4}}^{\prime}= & -13.5802+3.2945 w_{1}-0.2511 w_{1} w_{2}-0.2008 w_{1}^{2} \\
& +0.1078 w_{2}^{2}
\end{aligned}
$$

According to the above formulas and (5), the poisson model parameter is calculated. Using (10), the estimation model for failure probability of each weather area is finally obtained. Putting the forecast weather data into the estimation model, the failure probability of the transmission line can be predicted.

Based on the outage and weather data of the transmission line during August 2014 to December 2014, the estimation model is verified. Taking two transmission lines in weather area $\mathrm{WA}_{0}$ as an example, the lightning density and maximum wind speed in September 2014 are converted into weather intensity and substituted into the model. Comparison between the predicted failure probability and actual outage number is shown in Fig. 7. Line $X_{1}$ is the Zhuhuang No. 2 line in weather area $\mathrm{WA}_{2}$ and $\mathrm{WA}_{4}$, which

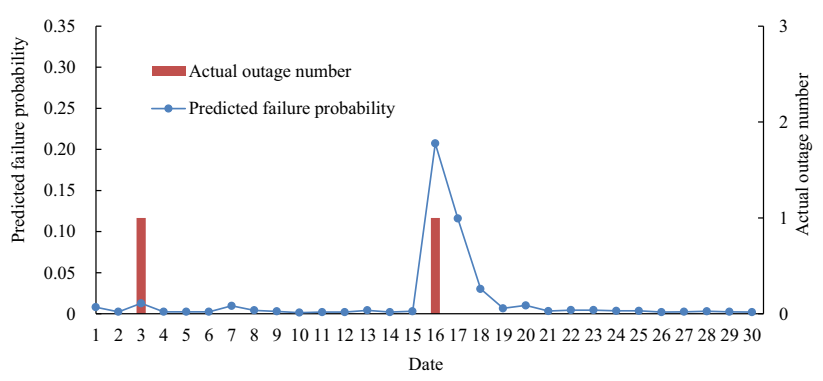

(a) Line $X_{1}$

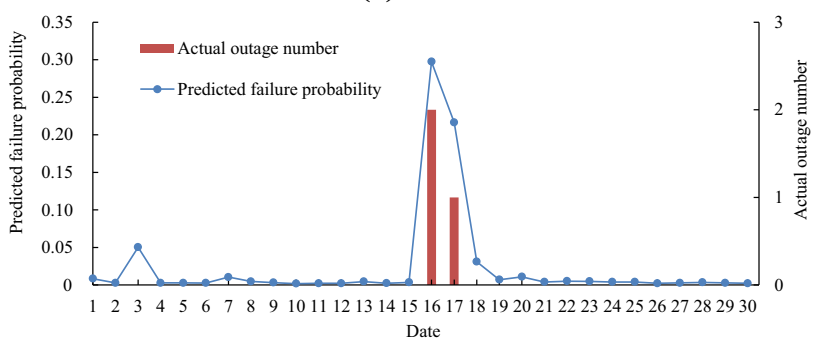

(b) Line $X_{2}$

Fig. 7 Comparison between the predicted failure probability and the actual outage number 
is $30.73 \mathrm{~km}$ long. Line $X_{2}$ is the Xinjiangshen line in $\mathrm{WA}_{1}$, $\mathrm{WA}_{2}$ and $\mathrm{WA}_{4}$, which is $60 \mathrm{~km}$ long. Weather area $\mathrm{WA}_{1}$ and $\mathrm{WA}_{4}$ are nearer to the seaside than weather area $\mathrm{WA}_{2}$. The minimum distances between these two lines and the seaside are all about $2 \mathrm{~km}$. Because line $X_{2}$ is longer and passes through weather area $\mathrm{WA}_{1}$, there is a longer line near the seaside. Most insulators used in these two lines are FXBW4-110/100.

Predicted failure probability from the estimation model is consistent with the actual outage events. When the predicted failure probability is high, the actual outage event happened. The predicted failure probability is largest on September 16, 2014 and it was the day when the typhoon "seagull" was striking the coast. In order to evaluate the accuracy of the prediction, predicted failure probability curves of two lines during August 2014 to December 2014 are presented in Fig. 8.

There is only one peak value more than 0.2 on the two curves of the predicted failure probability, which is during the typhoon "seagull." In the curve of line $X_{1}$, most of the predicted failure probability is less than 0.05 and the proportion is about $97.4 \%$. There was just one outage on September 3, 2014 with the predicted failure probability less than 0.1. The outage reason recorded is lightning. But the weather data shows very low lightning activity density on this day. There are two days during typhoon "seagull" with one outage and the predicted failure probability is more than 0.2. In the curve of line $X_{2}, 96.1 \%$ of the predicted failure probability is less than 0.05 . There is no outage with the predicted failure probability less than 0.1 . There are two days during typhoon "seagull" with two
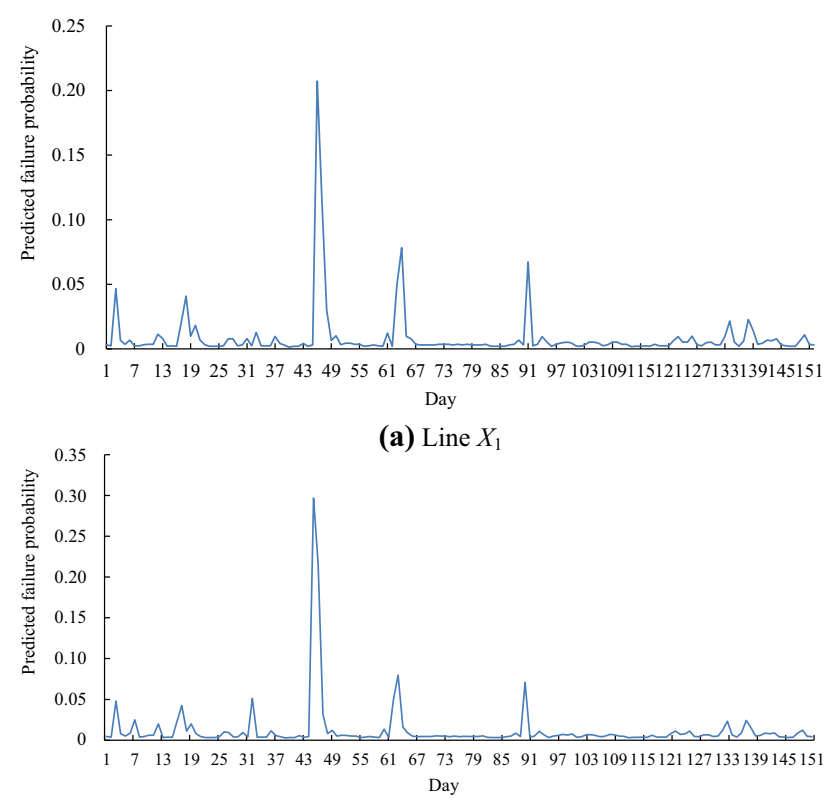

(b) Line $X_{2}$

Fig. 8 Predicted failure probability of two lines during August 2014 to December 2014 outages and the predicted failure probability is more than 0.3 . In general, the calculation model can reliably predict the outage probability of transmission lines. 0.2 can be set as a pre-warning value of outage probability for the power grid company.

\section{Conclusion}

1) Weather-related outage accounts for $78.09 \%$ according to the outage data analysis of the $110 \mathrm{kV}$ transmission line in the Guangxi Zhuang Autonomous Region of China during 2012-2014. The distribution of weatherrelated outages for time and space is consistent with the temporal and spatial variation of weather factors.

2) An estimation method for failure probability of transmission lines is proposed in this paper. It splits and aggregates the historical weather and outage data with an interval of one day for each weather area. The weather data is converted to weather intensity and aggregated as a weather block. The statistical characteristics of the outage event in each weather block are investigated based on the poisson model. The relationship between the poisson model parameter and weather data is analyzed via regression analysis.

3) The example shows that the model can reliably predict the failure probability of transmission lines. It can effectively reflect the influence of a typhoon. According to the weather forecast data, the power grid company could predict the failure probability for each weather area using this method. 0.2 can be set as a pre-warning value of the outage probability. Then the power grid company can send operational staff to the area with the highest probability, especially more than 0.2 . When there is an outage of transmission lines caused by severe weather, the repair time could be reduced.

Acknowledgements This work is supported by National Natural Science Foundation of China (No. 51307125).

Open Access This article is distributed under the terms of the Creative Commons Attribution 4.0 International License (http:// creativecommons.org/licenses/by/4.0/), which permits unrestricted use, distribution, and reproduction in any medium, provided you give appropriate credit to the original author(s) and the source, provide a link to the Creative Commons license, and indicate if changes were made.

\section{References}

[1] Balijepalli N, Venkata SS, Richter CW et al (2005) Distribution system reliability assessment due to lightning storms. IEEE Trans Power Deliv 20(3):2153-2159 
[2] Li G, Zhang P, Luh PB et al (2014) Risk analysis for distribution systems in the northeast US under wind storms. IEEE Trans Power Syst 29(2):889-898

[3] Kouassi A, Bourinet JM, Lalléchère S et al (2016) Reliability and sensitivity analysis of transmission lines in a probabilistic EMC context. IEEE Trans Electromagn Compat 58(2):561-572

[4] Ruszczak B, Tomaszewski M (2015) Extreme value analysis of wet snow loads on power lines. IEEE Trans Power Syst 30(1):457-462

[5] Billinton R, Acharya JR (2006) Weather-based distribution system reliability evaluation. IEE Proc Gener Transm Distrib 153(5):499-506

[6] Bhuiyan MR, Allan RN (1994) Inclusion of weather effects in composite system reliability evaluation using sequential simulation. IEE Proc Gener Transm Distrib 141(6):575-584

[7] Ward DM (2013) The effect of weather on grid systems and the reliability of electricity supply. Clim Change 121(1):103-113

[8] Billinton R, Cheng L (1986) Incorporation of weather effects in transmission system models for composite system adequacy evaluation. IEE Proc Gener Transm Distrib 133(6):319-327

[9] Billinton R, Singh G (2006) Application of adverse and extreme adverse weather: modelling in transmission and distribution system reliability evaluation. IEE Proc Gener Transm Distrib 153(1):115-120

[10] Wang P, Billinton R (2001) Reliability cost/worth assessment of distribution systems incorporating time-varying weather conditions and restoration resources. IEEE Trans Power Deliv 17(1):260-265

[11] Bollen MHJ (2001) Effects of adverse weather and aging on power system reliability. IEEE Trans Ind Appl 37(2):452-457

[12] Wang C, Hou Y, Qin Z et al (2015) Dynamic coordinated condition-based maintenance for multiple components with external conditions. IEEE Trans Power Deliv 30(5):2362-2370

[13] Arab A, Tekin E, Khodaei A et al (2016) System hardening and condition-based maintenance for electric power infrastructure under hurricane effects. IEEE Trans Reliab 65(3):1457-1470

[14] Liu Y, Singh C (2010) Reliability evaluation of composite power systems using Markov cut-set method. IEEE Trans Power Syst 25(2):777-785

[15] Caswell HC, Forte VJ, Fraser JC et al (2011) Weather normalization of reliability indexes. IEEE Trans Power Deliv 26(2):1273-1279

[16] Wang J, Xiong X, Zhou N et al (2016) Time-varying failure rate simulation model of transmission lines and its application in power system risk assessment considering seasonal alternating meteorological disasters. IET Gener Transm Distrib 10(7): $1582-1588$

[17] Panteli M, Mancarella P (2015) Influence of extreme weather and climate change on the resilience of power systems: impacts and possible mitigation strategies. Electr Power Syst Res 127:259-270

[18] Schlapfer M, Mancarella P (2011) Probabilistic modeling and simulation of transmission line temperatures under fluctuating power flows. IEEE Trans Power Deliv 26(4):2235-2243

[19] Billinton R, Li WY (1991) A novel method for incorporating weather effectsin composite system adequacy evaluation. IEEE Trans Power Syst 6(3):1154-1160

[20] Panteli M, Mancarella P (2017) Modeling and evaluating the resilience of critical electrical power infrastructure to extreme weather events. IEEE Syst J 11(3):1733-1742

[21] Alvehag K, Soder L (2011) A reliability model for distribution systems incorporating seasonal variations in severe weather. IEEE Trans Power Deliv 26(2):910-919

[22] Zhou Y, Pahwa A, Das S (2004) Prediction of weather-related failures of overhead distribution feeders. Prob Eng Inf Sci 20(1):959-962
[23] Li W, Zhou J, Xiong X (2008) Fuzzy models of overhead power line weather-related outages. IEEE Trans Power Syst 23(3):1529-1531

[24] Liu Y, Singh C (2010) Evaluation of hurricane impact on composite power systemreliability considering common-cause failures. Int J Syst Assur Eng Manag 1(2):135-145

[25] Liu Y, Singh C (2011) A methodology for evaluation of hurricane impact on composite power system reliability. IEEE Trans Power Syst 26(1):145-152

[26] Zhou Y, Pahwa A, Yang S (2006) Modeling weather-related failures of overhead distribution lines. IEEE Trans Power Syst 21(4):1683-1690

[27] Chen J, Zhao C, Gu S et al (2016) Present status and development trend of lightning detection and protection technology of power grid in China. High Volt Eng 42(11):3361-3375

Shuai YANG received the B.Sc. degree in Electrical Engineering and Automation from Wuhan University, in 2009, and the Ph.D. degree in High Voltage and Electrical Insulation from Wuhan University, Wuhan, China, in 2014. Currently, he is a post-doctor in Wuhan University. His research is mainly focused on electrical insulation.

Wenjun ZHOU received the Ph.D. degree in 1990 from Wuhan University of Hydraulic and Electrical Engineering. Currently, he is a professor at the School of Electrical Engineering of Wuhan University, China. He is a member of the High Voltage Committee of the Chinese Society of Electrical Engineering (CSEE), the Electrotechnical Test and Measurement Committee of the China Electrotechnical Society (CES), and the China Lightning Protection Standard Committee. He is also the vice director of the Hubei High Voltage Committee. His research interests include lightning protection and the diagnostic techniques for outdoor electrical insulations.

Shiyang ZHU received the Master degree in Electrical Engineering from Wuhan University, Wuhan, China in 2006. He has worked at Electric Power Research Institute, Guangxi Power Grid Co., Ltd., Nanning, China since 2006. His major research fields are high voltage technology and transmission line management.

Le WANG received the Master degree in Electrical Engineering from Guangxi University, Nanning, China in 2012. She has worked at Electric Power Research Institute, Guangxi Power Grid Co., Ltd., Nanning, China since 2012. Her major research fields are condition monitoring and disaster prevention and mitigation of transmission facility.

Lisha YE received the Master degree in Electrical Engineering from Wuhan University, Wuhan, China in 2016. She has worked at PowerChina Hubei Electric Engineering Corporation, Wuhan, China since 2016. Her major research field is design of transmission line.

Xuezhi XIA is pursuing for the Master degree in Electrical Engineering at Wuhan University, Wuhan, China since 2015. Her major research field is lightning protection grounding technology.

Han LI received his B.Sc. degree from the School of Electrical Engineering, Wuhan University, China in 2002, and M.Sc. degree from South China University of Technology in 2006, and the Ph.D. degree from Wuhan University in 2011. He is now a lecturer at the School of Electrical Engineering, Wuhan University. His research is mainly focused on high voltage and electrical insulations. 